\title{
NASA's Myriad uses of Digital Video
}

Rodney Grubbs

NASA DTV Program Lead

Marshall Space Flight Center, AL

Walt Lindblom

DTV Project Manager

Computer Sciences Corporation

Huntsville, AL

Sandy George

DTV Senior Engineer

Computer Sciences Corporation

Huntsville, AL

\section{Abstract}

Since it's inception, NASA has created many of the most memorable images seen this Century. From the fuzzy video of Neil Armstrong taking that first step on the moon, to images of the Mars surface available to all on the internet, NASA has provided images to inspire a generation, all because a scientist or researcher had a requirement to see something unusual.

Digital Television technology will give NASA unprecedented new tools for acquiring, analyzing, and distributing video. This paper will explore NASA's DTV future.

The agency has a requirement to move video from one NASA Center to another, in real time. Specifics will be provided relating to the NASA video infrastructure, including video from the Space Shuttle and from the various Centers.

A comparison of the pros and cons of interlace and progressive scanned images will be presented.

Film is a major component of NASA's image acquisition for analysis usage. The future of film within the context of DTV will be explored. 


\section{Introduction}

If one was asked to name the most memorable moments of the US Space Program, images instantly come to mind. Answers might include: the shadowy image of Neil Armstrong taking his first step on the moon, the instrumentation ring of the Saturn V rocket separating with the Earth in the background, John Young taking the Lunar Rover out for a spin on the moon, the first images of the Earth from the moon during Apollo 8, the launch of the lunar module from the moon, the first untethered space walk from the Space Shuttle, three space-suited astronauts grabbing a satellite with their hands in the cargo bay of the Space Shuttle, and the list might go on and on.

While NASA's critics might complain that the Agency spends too much time and money doing public relations, the reality is most, if not all, of these images were obtained using systems designed to aid scientists, researchers, and engineers. The need to see what can't be seen by human eyes drives NASA to build and deploy camera systems in some of the most unlikely of places. Every time the Space Shuttle launches, more than 100 film and video cameras record the event. Some of the cameras are used by engineers to monitor a component's performance during launch. Others are looking for propellant leaks and other safety related requirements.

Currently, there are two ways for the public to receive NASA's video and film imageryvia NASA TV, or by requesting it via the Freedom of Information Act. NASA TV is the primary way the media receives video from NASA. When there isn't a manned space mission underway, NASA uses one of it's two commercial satellite transponders to provide content for media stories, called video files, that include sound bites and b-roll explaining a particular discovery or technology. NASA also provides live interviews with researchers and scientists directly to individual television stations. During manned space missions NASA uplinks live video from space, coverage from mission control, and media opportunities and press briefings. In all cases, the video is provided "raw", to allow broadcasters and non-broadcasters the opportunity to edit the footage and provide their own supers and logo insertions. Requesting film, video, or photographs through the Freedom of Information Act is a much more laborious process, and requires the requestor to reimburse the government for any reproduction and shipping costs.

NASA is now working to transition its infrastructure for the digital world. The Space Act that created NASA in 1958 calls for NASA to provide dissemination of information about its activities in the most practical method possible. For years, television has been the best way for NASA to fulfill this charge. In the future, NASA hopes to take advantage of digital technologies to continue to provide memorable recordings of discoveries and endeavors in space. More importantly, though, this new technology will enhance the ability of NASA scientists, researchers, and engineers to conduct their research and monitor experiments.

\section{NASA and DTV Technologies}

There are several areas where digital television technologies enhance NASA's missions. NASA, like other entities that distribute video, has a limited bandwidth capacity through 
which to transport video, audio, and data. Downlinks from the space shuttle are currently limited to $50 \mathrm{Mbps}$, with a payload capacity of around $43 \mathrm{Mbps}$. Transmission capabilities within NASA includes satellite links, ATM, and in some cases, Next Generation Internet. Therefore, high quality compression of video is crucial to the continued success of NASA's highly advanced research.

High quality image capture is also a requirement. Getting into orbit is very expensive, so having high quality video transmitted to the ground can save money and lives should something go wrong with an experiment or with a space vehicle. There have been occasions where video was the only way to determine whether an experiment in the shuttle succeeded or failed. Every launch is monitored in real time and analyzed afterward by utilizing video and film from high-speed cameras. Debris, leaks, and anomalies with the propulsion systems are examples of what is looked for after every launch. Before an advancement to the Shuttle is flown, hours of ground testing is completed at NASA Centers. Video and high-speed film (usually transferred to video) is used to analyze the performance of engine components or space flight hardware. When film isn't practical or possible, it becomes very important that video imagery obtained is capable of extracting detailed, consistent information. Digital video technology provides excellent capabilities for NASA to provide high quality motion imagery that is useful to scientists, researchers, and engineers, while also fulfilling the mission to disseminate that video to the public via the media.

Since much of what researchers are looking for is not only finite and detailed, but also something that happens in less than a second, high frame rates are often required. For that reason, high-speed film will continue to be the image capture format of choice for such things as launch analysis. The advent of advanced telecines that have the ability to capture film frames as data dramatically increases the value of the film as an analysis tool.

Each of these non-broadcast uses of video by NASA will be explored in more detail:

\section{Distribution}

NASA's existing television broadcast system utilizes satellite and terrestrial links. NASA has two satellite transponders on GE Spacenet 2 that are used for broadcasting video and data. Transponder 5 is used primarily for internal distribution of video and data between centers. Transponder 9 is used to send video to media outlets and other NASA centers as required. Terrestrial links are also used to facilitate video transfer between NASA Centers. The terrestrial links are via NASA's Wide Area Network (WAN). This network is referred to as the NASA Integrated Service Network or "NISN". The NISN Backbone currently uses a Sprint ATM backbone infrastructure. NASA sites have OC3 or DS3 connectivity.

During a NASA mission, the current on-orbit configuration utilizes both transponders. The shuttle sends its signal to the TDRSS satellite. The downlinked signals from the shuttle are received at the NASA Ground Terminal (NGT), located at White Sands, New 
Mexico. NGT receives the signal and re-transmits the signal via satellite transponder 5. The signals from transponder 5 are then downlinked at Johnson Space Center (JSC) and Marshall Space Flight Center (MSFC). At JSC, the signal is demultiplexed into individual data and video streams and sent to other NASA centers or scientific research contacts as requested. JSC and MSFC transmit the video via transponder 9 for reception by the News Media. Figure 1 depicts this configuration.

\section{Shuttle Video/Data Current On-Orbit Configuration}

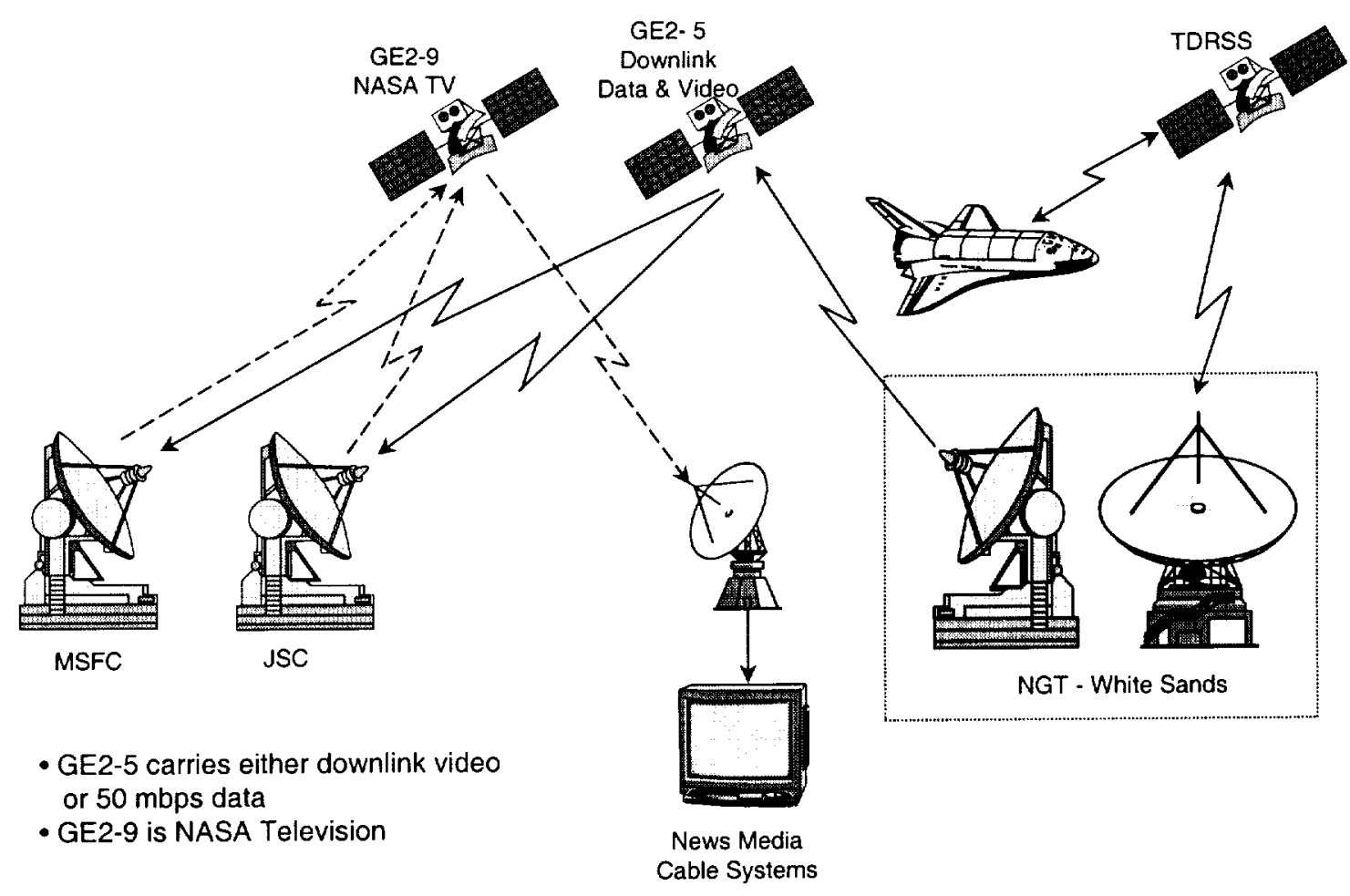

Figure 1 - Current On-Orbit Television Distribution Configuration

During a shuttle launch, two video signals are multiplexed at half frame rate. The two signals are used by propulsion engineers at MSFC to look for engine anomalies, and by shuttle engineers at JSC for readiness evaluation. The signals are referred to as ICE and MER video. They are carried by transponder 5. Public dissemination of NASA video by the media is very important to NASA in its objective to inform the public of its projects, goals, and objectives. The news media signal is carried over transponder 9. Figure 2 depicts this configuration. 


\section{Shuttle Video \\ Current Launch Configuration}

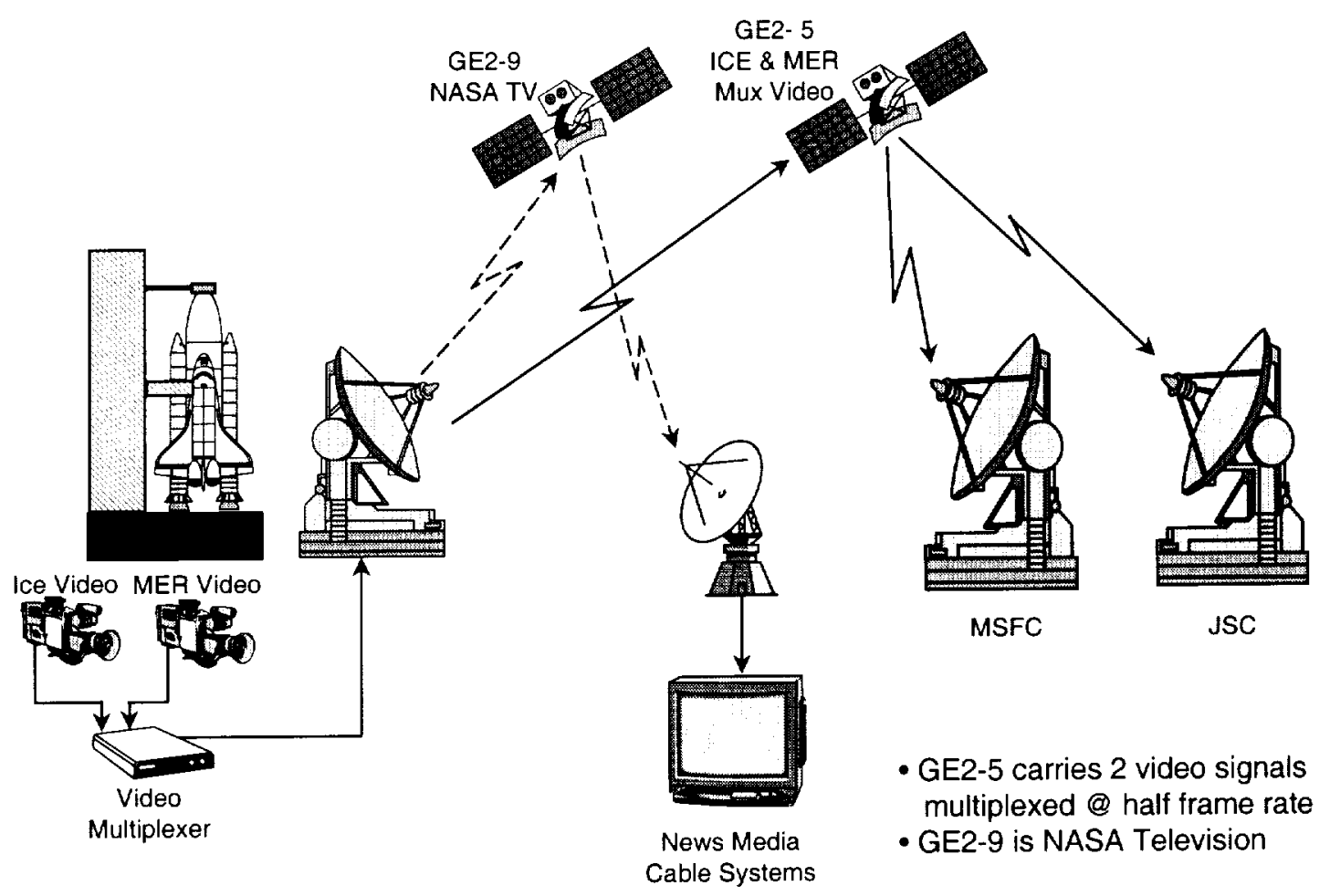

Figure 2 - Shuttle Launch Video Configuration

The use of quality compression techniques is crucial to NASA, especially in space, where limited bandwidth downlink capabilities exist. Video signals have to share limited bandwidth with experiment and telemetry data. During video compression, it is important for NASA not to experience data loss. This data is valuable and required for use by the NASA Scientists. The NASA DTV Working Group has conducted MPEG-2 compression testing with NASA Researchers and Developers across the agency. The test scenario examined six different data rates which ranged from 2 to $12 \mathrm{Mbps}$ and at three different GOP (groups of pictures) which ranged from 1 GOP (all I frames) to 16 GOP The MPEG test was configured using Betacam SP videotape format VTRs for playback and recording. The playback signal was routed as NTSC composite video into an MPEG- 2 codec. The coding structures were adjusted and optimized to enhance temporal resolution, enhance spatial resolution, and locate a median point between temporal and spatial resolution. The encoded/decoded video was output as NTSC composite video and recorded. The audio signal was also routed through the codec and encoded at $128 \mathrm{Kbps}$, Musicam format. The majority of the scientists were satisfied with $8 \mathrm{Mbps}$ or higher at either 8 or 16 GOP. Figure 3 shows how the test scenario was configured. This testing laid groundwork for evaluating future acceptable compression levels to support NASA's science community. 


\section{DTV MPEG Test System Configuration}

Play back VCR

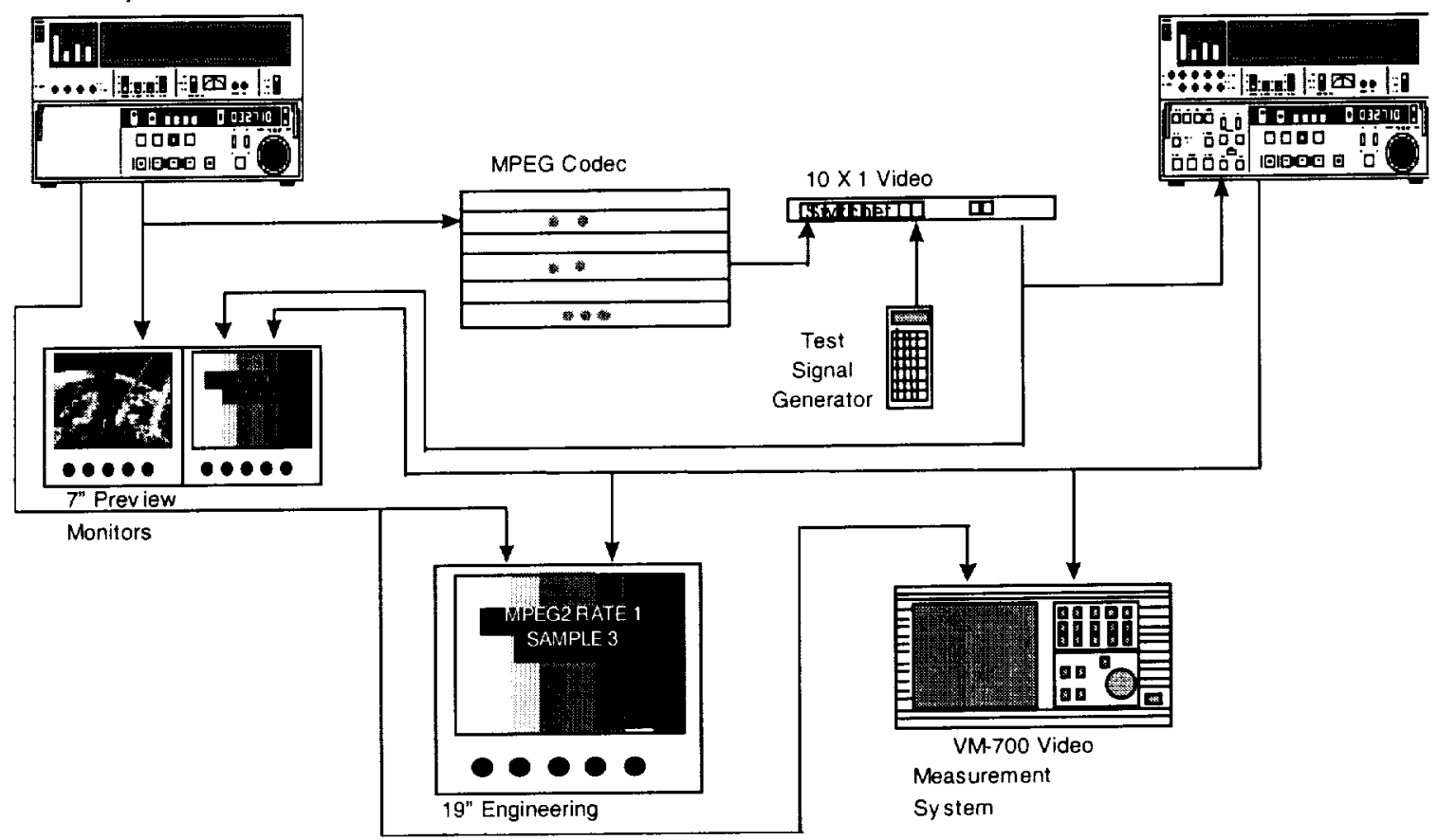

Figure 3 - MPEG Test Configuration

NASA is continuing to examine industry initiatives to improve video compression algorithms in order to improve throughput and performance. NASA feels that enhancements in this area will be key to reducing the cost of transmitting video while maintaining or improving levels of service.

NASA will still utilize satellite transmission to support needed video multicast transmission requirements until terrestrial service offerings are available that provide needed capabilities at a lower cost with comparable or greater reliability. NASA optimistically continues to examine new video transmission service and technology offerings that will benefit its goal to disseminate video of all program activities. Figure 4 depicts the possible on-orbit configuration that NASA is working toward in the near future. 


\section{Shuttle Video/Data Possible On-Orbit Configuration}

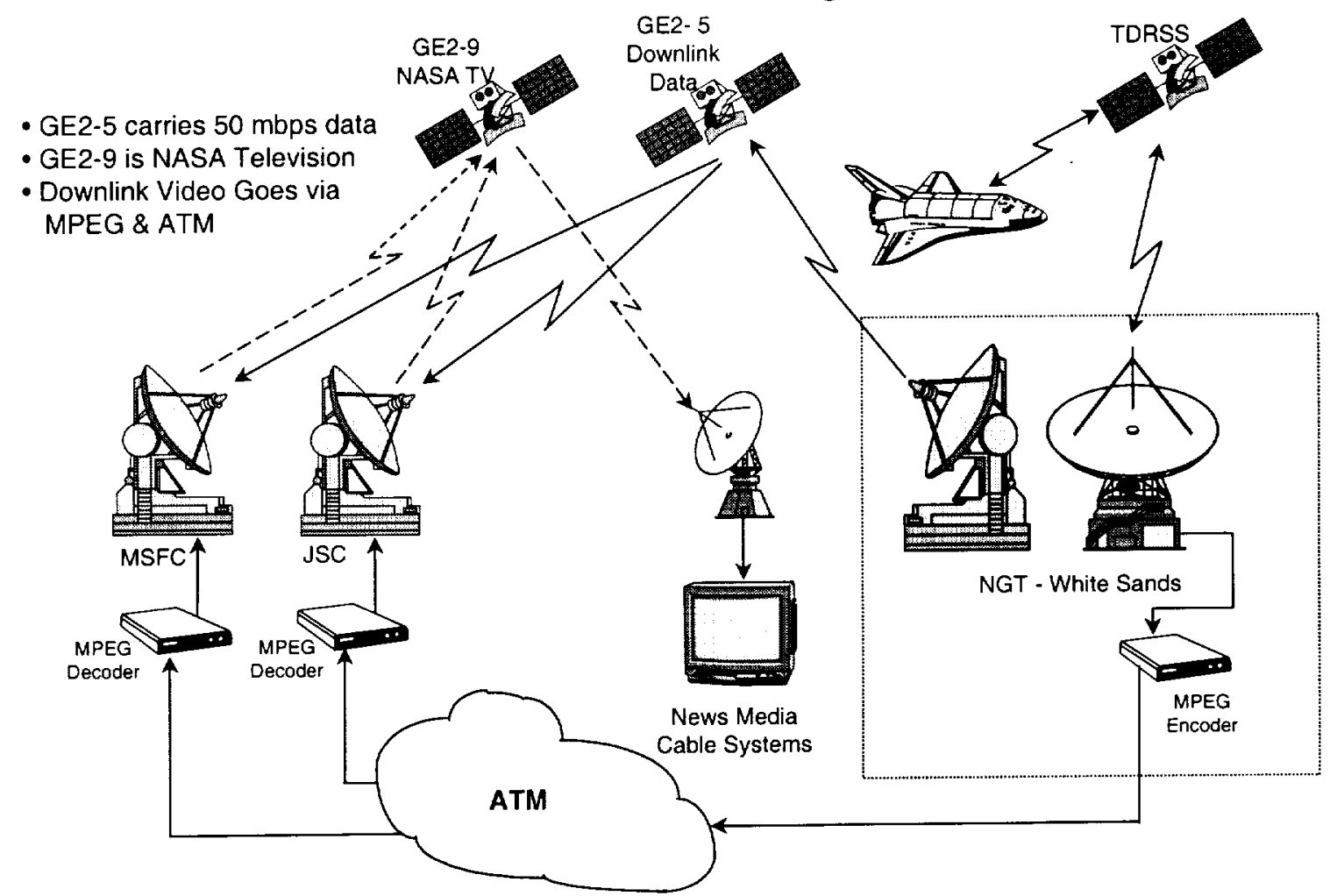

Figure 4 - Analog/Digital Hybrid Distribution

NASA's future and long term goal for distributing NASA video is to eliminate "tape" copies by sending electronic files. In this mode, the video files would be placed on a video server and customers would access the files via direct server connectivity or an URL connection.

\section{Digital Video Imagery}

In the analog television environment, video has played an important role within NASA for verification of events. But, due to the limitations of analog, video has not typically been used as a measurement tool. With square pixels and the ability to transfer images directly into computers, digital television is providing new tools for NASA researchers.

As it is in many organizations, the migration to digital video within NASA has been slow. Each of the NASA field centers has independent budgets for video systems upgrades. There are a few centers with digital video capability, but the majority of NASA centers still face the transition to digital. Because of this, NASA has a unique opportunity to implement digital video systems that best fit requirements for analysis, operations, and public release. In many cases, this will be a mix of Standard Definition Television (SDTV) and High Definition Television (HDTV). Not all requirements need HDTV. Monitoring ice build-up on a fuel line prior to a shuttle launch, for example, does not need a great deal of detail. Monitoring the start of the Space Shuttle engines is 
much more critical. Often problems that occur during engine start-up are detected by instruments and leave no trace except for telemetry data and the images captured by film or video. This imagery is of vital importance in the diagnosis of problems.

One area of consensus within the NASA video community is there is little room for multiple video standards when video is to be interchanged between the centers. To determine the standards to be used, a NASA-wide standards group, part of the NASA DTV Working Group, has been formed. This group has the responsibility to recommend acquisition, recording, and distribution standards to be adopted by NASA. As it will not be practical to implement multiple SDTV or HDTV standards, it is vital this group choose standards that provide the best overall video product for all requirements.

In SDTV, the standards group is recommending the use of 480P, 60 FPS, 16:9 format. It was felt by the group this standard would fulfill virtually all SDTV requirements. It can be transcoded to NTSC or HDTV, giving good results in either direction. Using 16:9 gives a wider field of view, which is important to research and operations users. It can be routed on SMPTE 259 systems. Recording systems are available and more are coming.

For HDTV, the choice was not as clear-cut. The major broadcast networks decision to choose different HDTV formats caused many in the standards group to doubt which format would be best for NASA. All HDTV formats are considered to have acceptable resolution for NASA requirements. Spatial resolution is important, but temporal resolution is more critical for research/analysis video. To help determine what choice to make, a comparison test for temporal resolution between interlace and progressive scan HDTV was conducted.

The test configuration used a prototype $720 \mathrm{p}$ portable camera, equipped with an $18 \times 7.8$ $\mathrm{HD}$ lens, and fiber optic connection to a CCU. A SMPTE-292 HD SDI output from the $\mathrm{CCU}$ was fed to a D5-HD VTR. Output of the VTR was fed to the A input of a digital waveform monitor.

The analog outputs of an HDCAM camcorder, equipped with an $15 \times 8 \mathrm{HD}$ lens, were fed via a coax bundle to an analog-to-digital converter. A handheld controller was used to control camera functions. The SMPTE-292 output of the A/D converter was fed to a D5-HD VTR. Output of the VTR was fed to the B input of the digital waveform monitor. It was confirmed by Sony a live camera feed from the analog outputs of the HDCAM are not filtered to 1440 pixels.

The digital waveform monitor was used for setup as well as a digital to analog converter. The analog outputs of the waveform monitor were fed to a 30 " HD monitor with 1000 TV lines of resolution. Figure 5 shows the test configuration. 


\section{Interlace/Progressive Test Configuration}

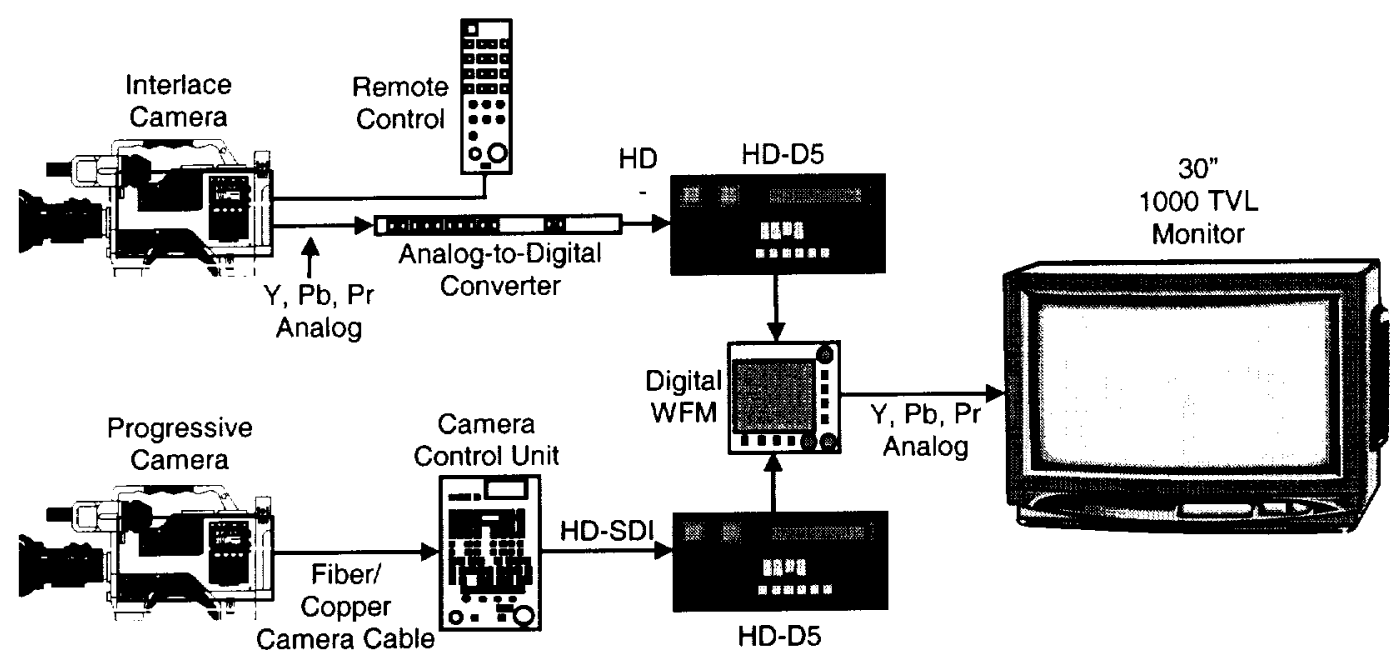

Figure 5 - Interlace/Progressive Test Configuration

The cameras were set-up by one engineer to be as closely matched as possible using the waveform monitor and the picture monitor. Parameters checked were black and white levels, gamma and knee settings, and detail level. Black and white levels were matched for each test object. All other set-up items were left at factory settings. Gamma, knee, and detail settings were matched on the resolution chart at the beginning of the test and not adjusted again.

Test material included the following:

- NTSC 4X3 Resolution Chart

- Vertical and Horizontal lines charts

- Line chart - 12 lines per inch on $81 / 2 \times 11$ paper

- Back Focus Wedge chart

- Text chart with various sizes of type

- $\$ 20$ bill

- Soccer ball

The line chart, back focus chart, and text chart were rotated on a turntable to simulate motion. The angular rate of motion was 3.34 degrees per field/frame at 59.94 FPS. All other material was shot static, with the exception of the soccer ball, which was bounced off the floor.

It was theorized before the test that distortions would be added to an interlaced picture by doing still images in field mode. Stills in this mode use a single field repeated to make a complete frame. This in effect does line doubling of the signal, which causes horizontal 
lines to be thicker than they should be. In practice, this was found to be the case. When using frame still mode on the VTR, interfield jitter precludes the use of the image for analysis. Video for analysis is used more as a series of stills than as motion video, which makes freeze frame performance critical.
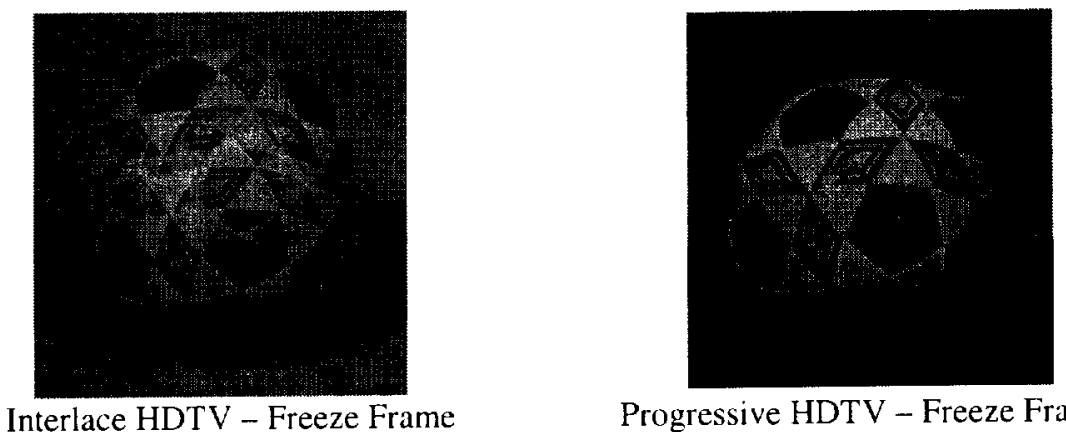

Interlace HDTV - Freeze Frame

Progressive HDTV - Freeze Frame

Progressive scan video does not have this problem. There was never a case where jitter or line doubling occurred. It should be noted that with either interlace or progressive, it is necessary to shutter the camera to achieve acceptable results with moving objects. Even at $1 / 1000$ of a second shutter speed, the outside edges of rotating charts were slightly blurry or showed some aliasing.

Of greatest interest was the performance of the cameras on high detail still images. It was theorized before the test that there would be little significant difference between the cameras, with the edge going to the interlace camera due to it's higher resolution imagers. This did not turn out to be the case.

On the resolution chart, both cameras showed 800 lines of resolution (the limit of the chart) in both horizontal and vertical, although only horizontal resolution was valid due to framing of the chart. The interlace camera had significant twitter on the horizontal wedges at 600 lines and above in frame or field freeze mode. The progressive camera showed no distortions in freeze frame.

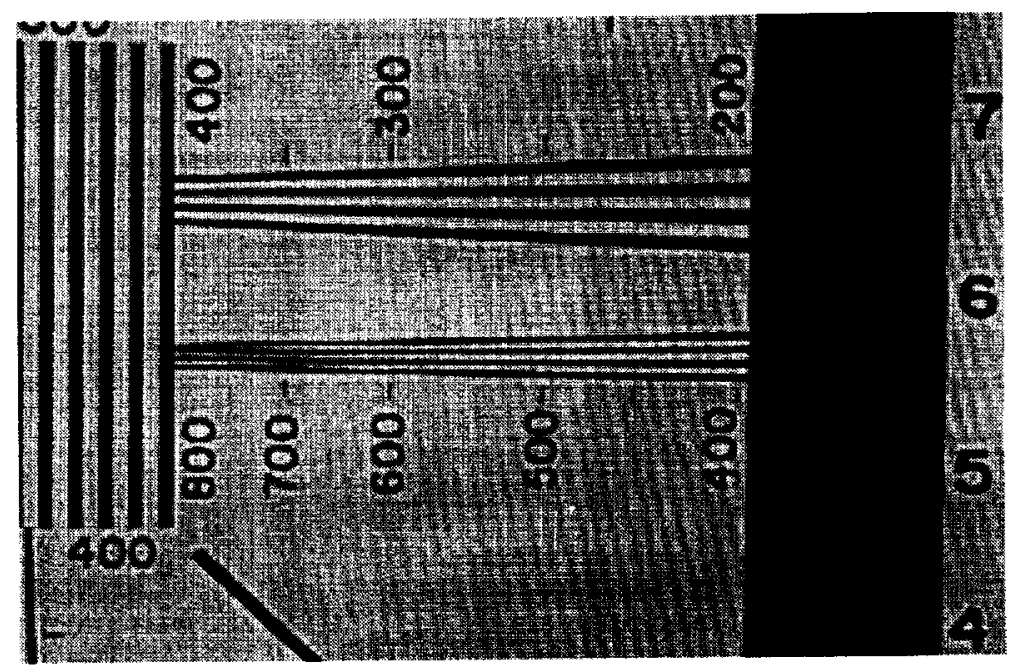

Interlace HDTV - Resolution Chart Horizontal Wedges 


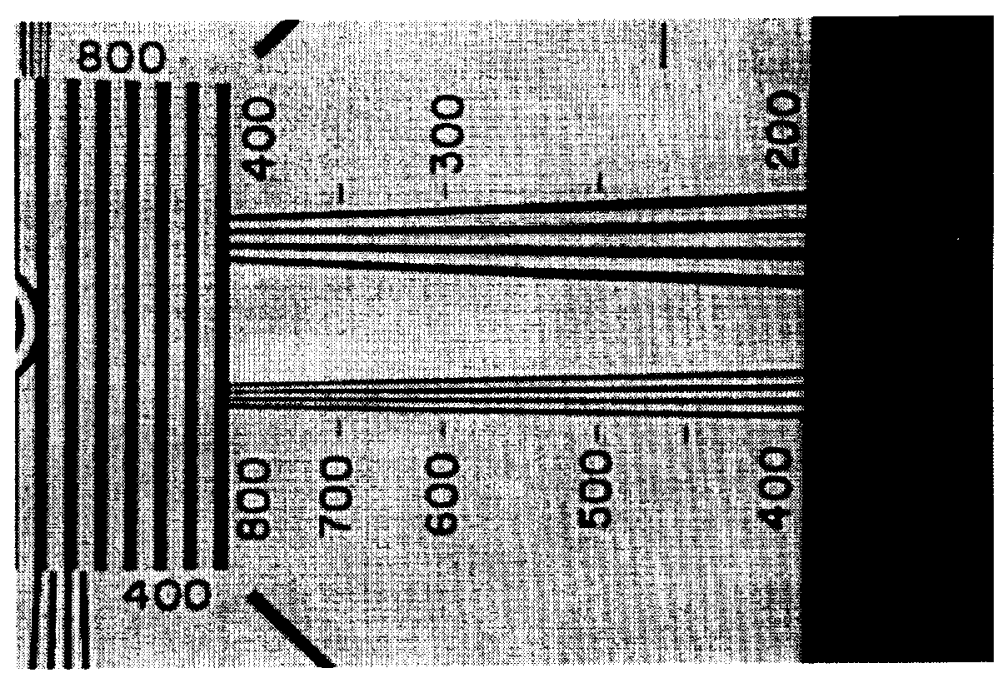

Progressive HDTV - Resolution Chart Horizontal Wedges

With the $\$ 20$ bill shot edge to edge, the engraving pattern behind the portrait of Andrew Jackson showed significant distortion on the interlace camera regardless of mode. In frame freeze, beat patterns formed chevrons in part of the pattern (this was partially due to the monitor). In field freeze, the curves in the lines of the pattern changed direction, from a downward curve to an upward curve. The progressive camera showed light beating in the pattern, resulting in a slight moiré effect (also partly due to the monitor). Aside from that, there were no distortions to the image.

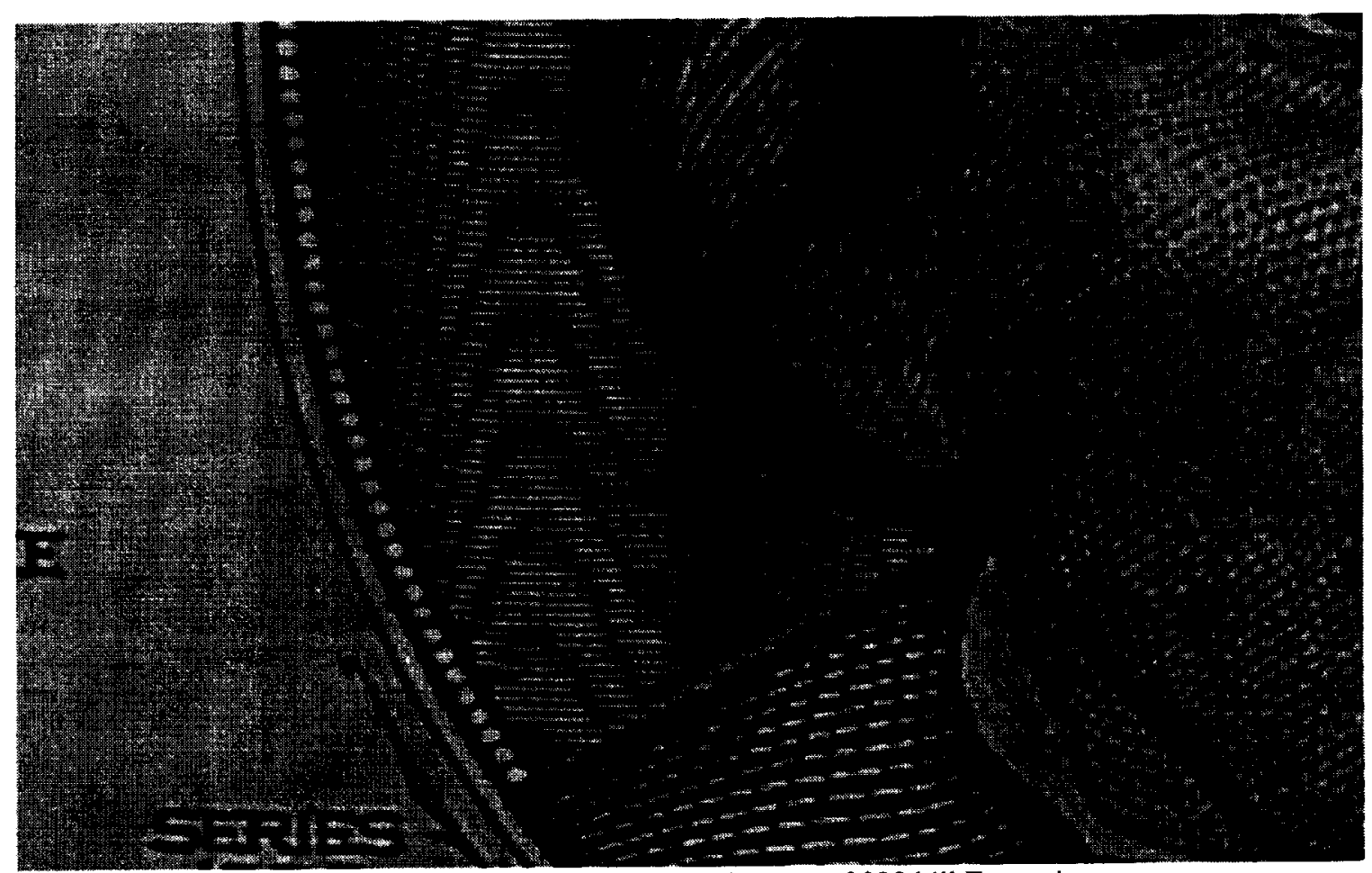

Interlace HDTV - Frame Freeze, Close-up of \$20 bill Engraving 


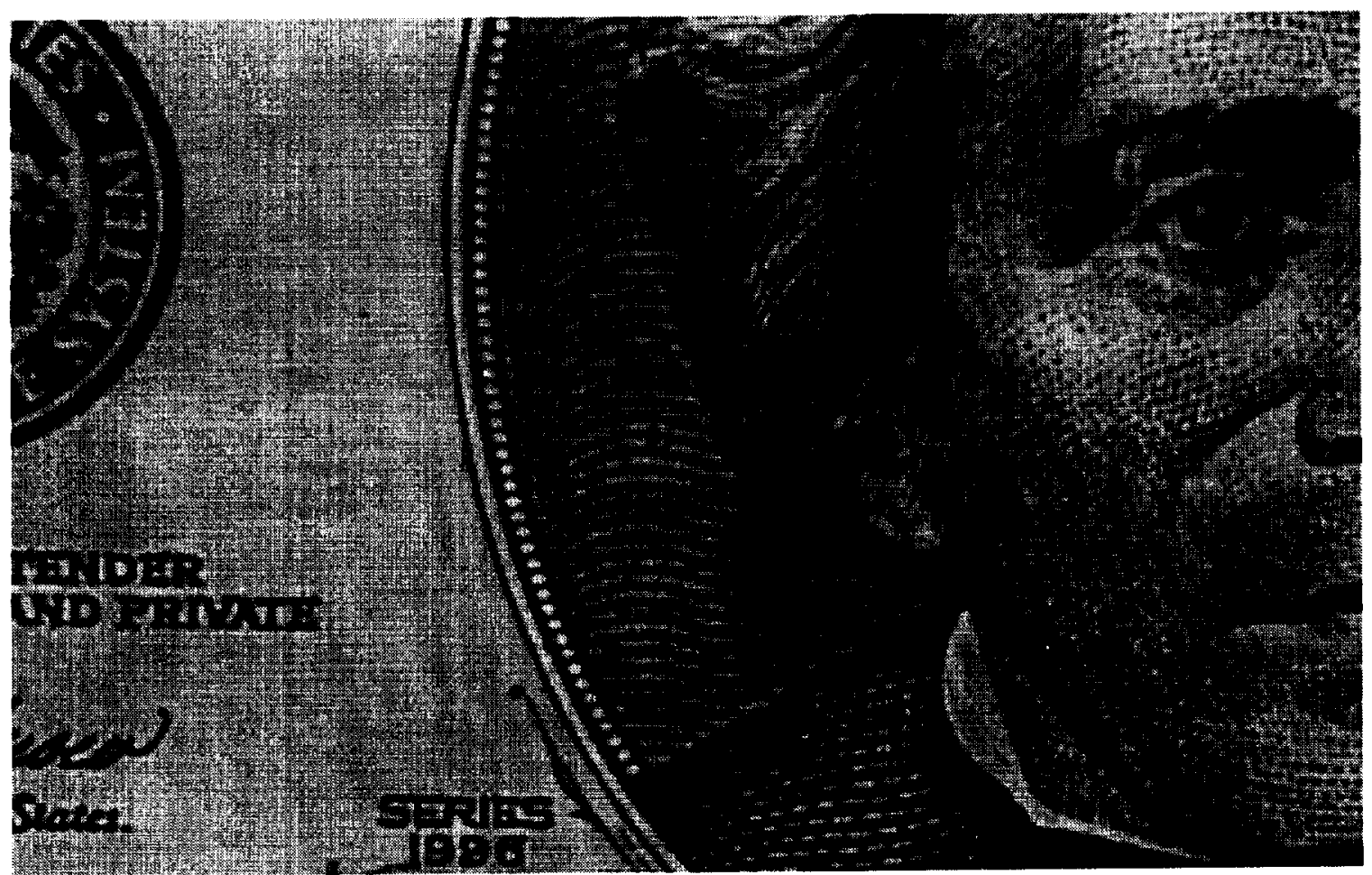

Interlace HDTV - Field Freeze, Close-up of \$20 Bill Engraving

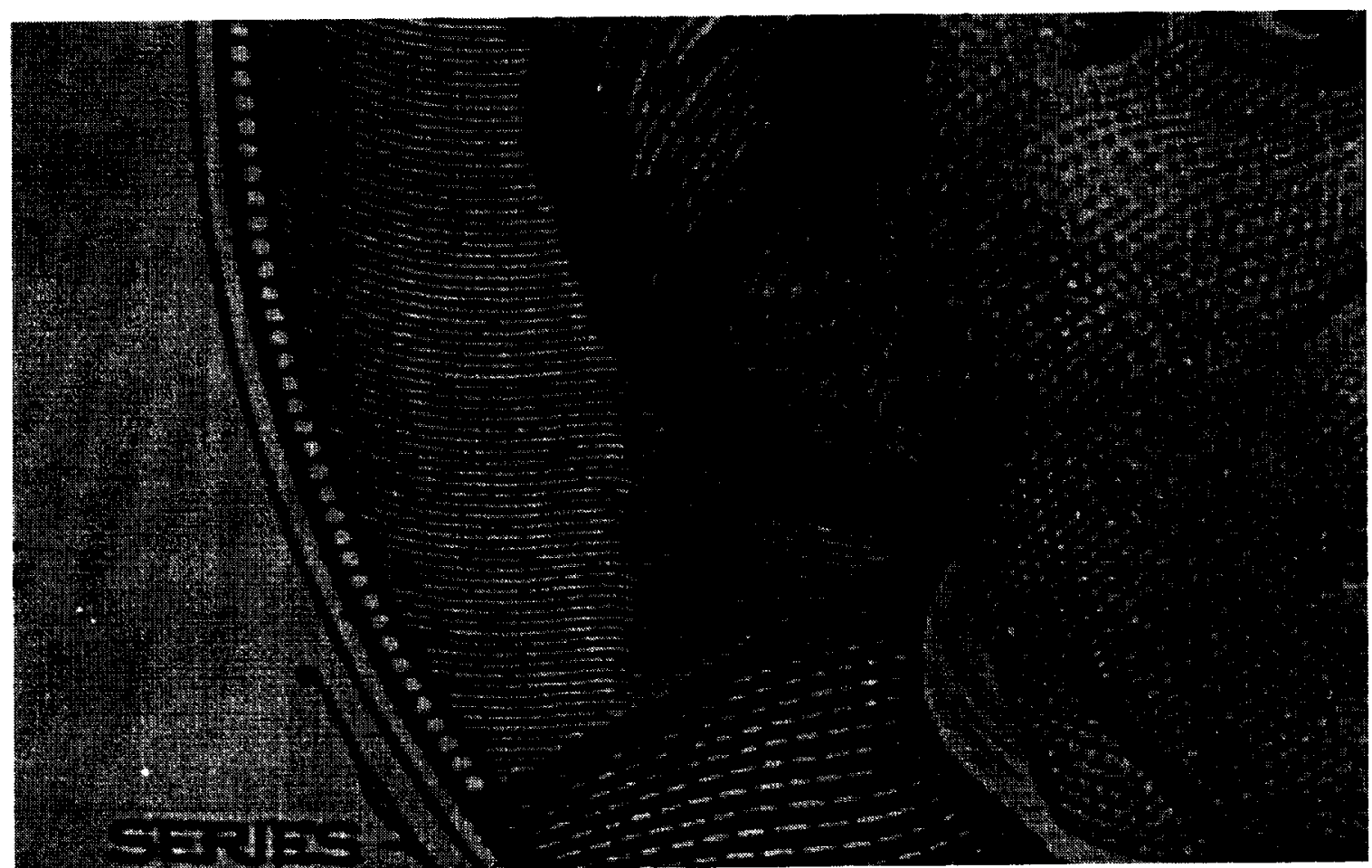

Progressive HDTV - Frame Freeze, Close-up of \$20 Bill Engraving 
It was concluded from the tests interlace video is not usable for analysis work of any video with motion. There are accuracy problems in the picture that prohibit the use of interlace video even when shooting still images of very fine detail.

Progressive scan video does not suffer from the same problems and provides pictures with a high degree of accuracy when compared to the original source.

Based on these tests, NASA is planning to implement progressive HDTV for all video to be used for analysis purposes. Use of interlace HDTV for other applications will be an interim solution until progressive equipment is readily available.

\section{Film and HDTV}

Every time the Space Shuttle lifts off there are at least 60 high-speed 16MM and 35MM cameras rolling. Some are documenting specific areas of a Space Shuttle Main Engine, some look at the bottom of a Solid Rocket Booster, and others track the entire vehicle throughout liftoff. Occasionally there are high-speed film cameras located in the nose cone of the solid rocket booster to document the parachute deploy during the descent of the booster back to the Atlantic Ocean. In all cases negative film is the medium of choice due to it's high resolution and ability to record events with a high frame rate. The average camera recording a launch films at 100 frames-per-second.

After a launch a team of film analysts watch each roll for any anomaly. Sometimes a video camera records something unusual, so the high-speed film from a camera with a similar view is used to help determine what the anomaly might be. Normally a special film projector is utilized that allows a specific frame from a film print to be observed on a screen without damaging the film. If further analysis is required, a film transfer to analog component video is provided, then selected frames are digitized using a mid-range computer.

In the future, specific frames of video will be converted directly as data for analysis. Tests have been conducted utilizing an HD telecine and color corrector. Frames from an A-wind first generation film print were color corrected and saved as $2 \mathrm{~K}$ by $2 \mathrm{~K}$ data files on a digital linear tape. Also, portions of selected frames were saved as $2 \mathrm{~K}$ by $2 \mathrm{~K}$ files. Not surprisingly, the images from 16MM frames were very grainy, particularly when a portion of the frame was digitized. Nevertheless, the image was sufficient to determine what occurred during that fraction of a second in time, in this case, the drogue shoot door hitting the end of a Main Engine during the launch of STS-95, John Glenn's return to space. In all cases, the image quality from $16 \mathrm{MM}$ and $35 \mathrm{MM}$ film frames using an $\mathrm{HD}$ telecine was far superior to any frame capture methods utilized currently by NASA.

Besides launches, NASA conducts research and testing at its field centers that require high-speed motion picture film. In some cases, such as explosive bolt testing, the event being recorded occurs in thousandths of a second. HD transfers at 30FPS eliminate $3: 2$ pull-down, allowing for frame by frame continuity. Conversion of frames or portions of frames directly to data, will greatly enhance the value of film as a recording medium for research and engineering. 


\section{Conclusion}

In the coming decade, NASA has plans to fly a remote piloted aircraft on Mars, capture and return comet dust, and explore ice caps on Europa, a moon of Jupiter. With DTV technology, images from missions such as these will take their place beside memorable images from NASA's past. At the same time, DTV technology will give researchers better tools to inspire a new generation of exploration. 